\title{
Preeclampsia como factor de riesgo independiente para el trastorno por déficit de atención con hiperactividad. Estudio de casos y controles. Bucaramanga, Colombia Preeclampsia as an independent risk factor for attention deficit hyperactivity disorder. Case control study. Bucaramanga, Colombia A pré-eclâmpsia como um factor de risco independente para o transtorno do déficit de atenção com hiperatividade. Estudo caso-control. Bucaramanga, Colômbia
}

\author{
Yuly Andrea Castellanos-Castellanos, MD*II \\ Mauricio Escobar-Sánchez, MD Psiq Infant** \\ María Carolina Páez-Leal, MD Msc*** \\ Luis Alfonso Díaz-Martínez, MD Msc****
}

\author{
Carlos Andrés Arias-Duran, MD* \\ Derlly Marcela Espitia-Orejarena, MD* \\ Jesica Lisette Forero-Parada, MD*
}

\section{Resumen}

Introducción: El trastorno por déficit de atención e hiperactividad es una condición mental que afecta a niños y adolescentes con prevalencia estimada de $5.3 \%$ en la población mundial y en Colombia es de $19-24 \%$ en hombres y $10-12 \%$ en mujeres. Su etiología es multifactorial, entre ellos se encuentra la hipoxia como factor del medio ambiente uterino; presentándose en entidades como la preeclampsia, donde se ha considerado determinante en el desarrollo del trastorno por déficit de atención e hiperactividad, siendo importante evaluar su asociación. Objetivo: Determinar la asociación entre el diagnóstico trastorno por déficit de atención e hiperactividad y el antecedente perinatal de preeclampsia. Metodología: Estudio de casos y controles retrospectivo de 411 casos de trastorno por déficit de atención e hiperactividad y 404 controles no emparentados sin trastorno ni otros trastornos disruptivos del comportamiento. La evaluación psiquiátrica se hizo entre el 2005-2011, mediante entrevista estructurada que incluyó indagar el antecedente de preeclamsia en el embarazo de cada paciente. Resultados: El antecedente de preeclampsia se presentó en el $3.7 \%$ de los pacientes con trastorno por déficit de atención e hiperactividad y en el 5.5\% de los controles (OR 0.66, IC95\% 0.34-1.29; $p=0.221$ ). Esta falta de asociación se mantuvo luego de ajustar por género, ser adoptado, haber nacido por cesárea y edad de inicio del trastorno que llevó a consultar al paciente (OR 0.51, IC95\% $0.22-1.17 ; p=0.115)$. Conclusión: El estudio no encontró significancia estadística entre el antecedente de preeclampsia materna y el trastorno por déficit de atención e hiperactividad como factor principalmente asociado en la muestra de pacientes seleccionados. [CastellanosCastellanos YA, Escobar-Sánchez M, Páez-Leal MC, DíazMartínez LA, Arias-Duran CA, Espitia-Orejarena DM, ForeroParada JL. Preeclampsia como factor de riesgo independiente para el trastorno por déficit de atención con hiperactividad. Estudio de casos y controles. Bucaramanga, Colombia. MedUNAB 2014; 17(1):23-29]

Palabras clave: Preeclampsia; Trastorno por déficit de atención con hiperactividad; Hipoxia encefálica.

\footnotetext{
* Medico, Facultad de Ciencias de la Salud, Universidad Autónoma de Bucaramanga, Floridablanca, Santander, Colombia.

** Médico, Especialista en Psiquiatría Infantil y del Adolescente, Grupo de Estudio Genético de Enfermedades Complejas, Centro de investigaciones Biomédicas, Docente, Programa de Medicina, Facultad de Ciencias de la Salud, Universidad Autónoma de Bucaramanga, Floridablanca, Santander, Colombia *** Médico, Magister en Biología Inmunogenética, Grupo de Estudio Genético de Enfermedades Complejas, Centro de Investigaciones Biomédicas, Docente, Programa de Medicina, Facultad de Ciencias de la Salud, Universidad Autónoma de Bucaramanga, Floridablanca, Santander, Colombia

****Médico, Especialista en Pediatría, Magister en Epidemiología, Profesor Titular, Escuela de Medicina, Facultad de Salud, Universidad Industrial de Santander, Bucaramanga, Colombia

I Estudiante de la Especialización de Epidemiología, Facultad de Ciencias de la Salud, Universidad Autónoma de Bucaramanga, Floridablanca, Santander, Colombia
}

Correspondencia: Mauricio Escobar Sánchez, Médico especialista en psiquiatría infantil, Facultad de Ciencias de la Salud, Universidad Autónoma de Bucaramanga, Campus el Bosque, Calle 157 \# 19-55 (Cañaveral Parque), Floridablanca, Santander, Colombia. Email: mescobar@unab.edu.co. 


\section{Abstract}

Background: Attention deficit hyperactivity disorder is a mental disorder that affects children and adolescents. Its prevalence is $5.3 \%$ in the global population, in Colombia is $19-24 \%$ in men and $10-12 \%$ in women. It's etiology is multifactorial, including hypoxia as a factor of the uterine environment; performing in entities such as preeclampsia, has been considered in the development of attention deficit hyperactivity disorder, been important to evaluate the association. Objective: Determine the association between attention deficit hyperactivity disorder diagnosis and perinatal history of preeclampsia. Methodology: Retrospective case-control study. 411 cases with attention deficit hyperactivity disorder and 404 unrelated controls without psychiatric or other disruptive behavior disorders. The psychiatric evaluation was made between 2005-2011 by structured interview, which will assess the obstetric history of mothers in search of preeclampsia. Results: The history of preeclampsia occurred in $3.7 \%$ of patients with attention deficit hyperactivity disorder and $5.5 \%$ of controls (OR $0.66,95 \% \mathrm{Cl} 0.34-1.29, \mathrm{P}=0.221$ ). This lack of association remains after adjusting for gender, if adopted, would have been born by caesarean section and age of onset of the disorder that led to refer the patient (OR 0.51 , $95 \% \mathrm{Cl} 0.22-1.17, \mathrm{P}=0.115)$. Conclusions: The study did not find satatistical significance between maternal history of preeclampsia and attention deficit hyperactivity disorder as the main factor associated in the sample of selected patients. [Castellanos-Castellanos YA, Escobar-Sánchez M, Páez-Leal MC, Díaz-Martínez LA, Arias-Duran CA, Espitia-Orejarena DM, Forero-Parada JL. Preeclampsia as an independent risk factor for attention deficit hyperactivity disorder. Case control study. Bucaramanga, Colombia. MedUNAB 2014; 17(1):23-29]

Keywords: Pre-Eclampsia; Attention deficit disorder with hyperactivity; Hypoxia encefálica

\section{Introducción}

El trastorno por déficit de atención e hiperactividad (TDAH) es uno de los trastornos mentales que afecta con frecuencia a niños y adolescentes, caracterizado por la presencia predominante de inatención, hiperactividad e impulsividad, siendo más común en hombres que en mujeres en una proporción de 2:1 hasta 5:1. ${ }^{1}$ Se estima una prevalencia general de 5 a $10 \%$ en la población mundial, ${ }^{2}$ con una prevalencia promedio de $5.3 \%$. $^{3}$ En Colombia, se encontró una prevalencia mayor a lo reportado en la literatura, con una frecuencia de 19 a $24 \%$ para hombres y de 10 a $12 \%$ para mujeres. $^{4-7}$

La etiología del TDAH no está totalmente esclarecida. Se conoce que es multifactorial, existiendo factores biológicos,

\section{Resumo}

Introdução: O transtorno do déficit de atenção com hiperactividade é uma condicção mental que afeta crianças e adolescentes. A sua prevalencia é de $5.3 \%$ da população mundial, e na Colômbia a sua incidência é de $19-24 \%$ nos homens e $10-12 \%$ nas mulheres. Sua etiologia é multifatorial, incluindo hipóxia como um fator do ambiente uterino; apreentando se em entidades como a pré-eclâmpsia, onde é considerado crucial para o desenvolvimento do transtorno do déficit de atenção com hiperactividade, constituindo dados importantes para avaliar sua associação. Objetivo: Determinar a associação entre o diagnóstico do transtono do déficit de atenção com hiperactividade e a história perinatal de préeclâmpsia. Metodologia: Estudo caso-control retrospectivo. 411 casos, com transtorno do déficit de atenção com hiperactividade e 404 controles independentes sem distúrbios de comportamento disruptivo psiquiátricos ou outros. A avaliação psiquiátrica foi feita entre 2005-2011 por meio de entrevista estruturada, que incluía investigação da história obstétrica das mães, para verificar antecedentes de préeclâmpsia. Resultados: Histórico de pré-eclâmpsia foi detectado em $3.7 \%$ dos pacientes com transtorno do déficit de atenção com hiperactividade e em $5.5 \%$ dos controles (OU 0.66, IC 95\% 0.34-1,29; p=0.221). A falta de associação permanece após o juste pelo gênero, para ser adotado, nascer por cesariana e idade de início da doença que levou a consultar do paciente (OU 0.51, IC 95\% 0.22-1.17; $p=0.115$ ). Conclusões: $O$ estudo Não encontrau significancia estatística entre história materna de pré-eclâmpsia e transtorno do déficit de atenção com hiperactividade como o principal fator, asociado na amostra de pacientes selecionados. [CastellanosCastellanos YA, Escobar-Sánchez M, Páez-Leal MC, DíazMartínez LA, Arias-Duran CA, Espitia-Orejarena DM, ForeroParada JL. A pré-eclâmpsia como um factor de risco independente para o transtorno do déficit de atenção com hiperatividade. Estudo caso-control. Bucaramanga, Colômbia. MedUNAB 2014; 17(1):23-29]

Palavras-chave: Pré-eclâmpsia; Transtorno do deficit de atenção com hiperatividade; Hipóxia encefálica.

ambientales y psicológicos que contribuyen a su desarrollo. Adicionalmente, se ha relacionado de múltiples factores del período perinatal dentro de los culaes se encuentra, la prematuridad y el bajo peso al nacer. Se ha evidenciado que estos factores se han asociado a TDAH, hallazgo frecuente en los hijos de madres preeclámpticas. ${ }^{8-10}$

La preeclampsia (PE) es una enfermedad multifactorial caracterizada por disfunción endotelial generalizado, el cual causa disminución del flujo sanguíneo; manifestándose con los signos cardinales típicos, como la hipertensión y la proteinuria. " Esta patología gestacional es una de las principales causas de morbi-mortalidad materna y perinatal en el mundo. ${ }^{12}$ La prevalencia exacta aún se desconoce; sin embargo, Martin y colaboradores reportan una tasa de $18 \%$ en $\mathrm{PE}$ severa en una Cohorte retrospectiva. ${ }^{12-13}$ En una 
revisión sistemática, se presenta los datos de incidencia en mujeres embarazadas con hipertensión crónica, en donde la PE obtuvo un alta incidencia con un $25.9 \%$ (IC 95\% 21.0$31.5 \%)^{14}$

Se acepta actualmente que factores de riesgo tales como, exposición al tabaco, consumo de alcohol, prematuridad, bajo peso al nacer y estados de hipoxia/anoxia durante el embarazo, entre otros, contribuyen al desarrollo del TDAH. ${ }^{10,15-20}$ Los datos expuestos dan relevancia a los factores que alteran el medio ambiente uterino como factores causales en esta entidad, ya sea involucrándose en la alteración de las vías dopaminérgicas o en el desarrollo de estructuras cerebrales dopaminérgicas como la corteza prefrontal, el globo pálido y los ganglios basales. ${ }^{21-22}$ Por tal razón, es importante conocer el impacto de los antecedentes obstétricos maternos en el riesgo de desarrollar TDAH, y en particular de los trastornos hipertensivos del embarazo.

Dado que en la literatura, se demuestra cómo estos trastornos se asocian a disfunción endotelial con inicio de afectación en la unidad materno-fetal, para posteriormente generalizarse, y así provocar hipoxia fetal y materna $;^{23}$ el cual se ha asociado como factor de riesgo importante para el desarrollo futuro de TDAH. Por ende, el objetivo de este trabajo es determinar en nuestra población la posible relación entre el diagnóstico de TDAH y el antecedente perinatal de PE.

\section{Metodología}

Se llevó a cabo un estudio observacional retrospectivo de casos y controles. Se incluyeron todos los pacientes registrados en la base de datos perteneciente a la consulta privada de un médico especialista en psiquiatría infantojuvenil de Bucaramanga, Santander, Colombia. La población a estudio consultó entre las edades de 7 a 18 años, en el periodo de 2005 a 2011.

Se comparó 411 niños y adolescentes con TDAH frente a 404 controles de entre los demás pacientes de la misma consulta y seleccionados de manera aleatoria; pareados por género y edad. Los controles fueron tomados al menos de la misma edad y hasta 2 años más del respectivo caso. La muestra se calculó basándose que entre los controles existiese la misma prevalencia del antecedente de PE de la población general (10\%), con un OR estimado de 3, bajo los criterios de confianza del $95 \%$ y un poder del $80 \%$.

Para la selección de la muestra de los casos, se tuvo en cuenta que el paciente cumpliese con los criterios A, B, C, D y E del DSM-IV TR (tabla 1) para el diagnóstico de TDHA, evaluados en la consulta psiquiátrica mediante una entrevista estructurada realizada por un psiquiatra infantojuvenil certificado por la Asociación Colombiana de Psiquiatría (ACP). Los controles fueron pacientes de la misma consulta, con diagnóstico diferente a TDAH u otro trastorno del comportamiento disruptivo. Los participantes se seleccionaron al azar de entre todos los pacientes potenciales, teniendo en cuenta el apareamiento propuesto por género y edad. En ambos grupos se excluyeron a los pacientes con retraso mental.

Se revisaron las historias clínicas de todos los pacientes que ingresaron entre el 2005 al 2011, en relación a edad de consulta, sexo del paciente, grado de escolaridad, antecedente de ser adoptado, edad de inicio de los síntomas que motivaron la consulta, antecedentes familiares de enfermedades cardiovasculares o TDAH, antecedentes de $\mathrm{PE}$, trastornos mentales o infecciosos durante el embarazo, vía del parto, complicaciones perinatales del paciente y diagnósticos en la esfera mental confirmados por el médico tratante.

Una vez identificados todos los potenciales casos y controles, se digitó de manera independiente la información en una base de datos por duplicado de todas las historias clínicas en Microsoft Excel 2007, bases que se compararon a fin de detectar errores de digitación; los cuales se corrigieron al verificar en las historias clínicas.

Dado que se tenían 411 casos y 404 controles potenciales con toda la información necesaria para hacer el análisis, y que la edad de inicio de síntomas de todos los casos fueron inferiores a la de todos los controles; se decidió no hacer el muestreo propuesto y analizar todos los pacientes sin apareamiento alguno.

Esta estrategia permitió tener un poder de $98 \%$ para detectar las diferencias propuestas iniciales, o del $80 \%$ para detectar un OR de 2.24. El análisis se hizo por medio de Stata 12.1 (StataCorp, College Station, Tx, 2012) con el fin de comparar inicialmente las diferencias entre casos y controles con las pruebas $\chi^{2}, t$ de student o de Wilcoxon según la naturaleza de cada variable. Se aceptó como diferencias significativas aquellas con $\alpha<0.05$. Finalmente, se corrieron modelos de regresión logística para estimar la razón de disparidades ajustada (odds ratio - OR) de la asociación entre el diagnóstico de TDAH y el antecedente de embarazo con PE, donde se buscaba cuidar cuidando buscar el modelo que fuese más parsimonioso y que tuviera adecuada bondad de ajuste.

\section{Resultados}

Entre el 2005 y 2011 se atendieron 1600 pacientes en la consulta privada del especialista en psiquiatría infantojuvenil. De ellos, 815 cumplían criterios para ser elegibles, ya sea como casos (411) o controles (404). Se encontró más varones entre los casos que en los controles $(75.4 \%$ vs $50.7 \%, \mathrm{p}<0.001$ ), así como más pacientes con historia de haber sido adoptados $(7.3 \%$ vs $3.2 \%, \mathrm{p}=0.012)$. Igualmente, 
Tabla 1. Trastorno por déficit de atención con hiperactividad, según DSMIV-TR

\begin{tabular}{l|l}
\hline \multicolumn{1}{c}{$\mathbf{A}(\mathbf{1}) \mathbf{0}(\mathbf{2})$} \\
\hline \multicolumn{1}{c}{1} & \multicolumn{1}{c}{2} \\
\hline $\begin{array}{l}\text { Seis o más de los siguientes síntomas han } \\
\text { persistido por lo menos durante 6 meses, con } \\
\text { intensidad que es desadaptativa e incoherente } \\
\text { con relación al nivel de desarrollo. }\end{array}$ & $\begin{array}{l}\text { Seis o más de los siguientes síntomas de } \\
\text { hiperactividad-impulsividad han persistido } \\
\text { durante por lo menos 6 meses, con una } \\
\text { intensidad desadaptativa e incoherente para el } \\
\text { nesatención: }\end{array}$
\end{tabular}

\section{Hiperactividad:}

a) A menudo no presta atención suficiente a los detalles o incurre en errores por descuido en las tareas escolares, en el trabajo 0 en otras actividades.

b) A menudo tiene dificultades par a mantener la atención en tareas o en actividades lúdicas.

c) A menudo parece no escuchar cuando se le habla directamente.

d) A menudo no sigue instrucciones y no finaliza tareas escolares, encargos, $u$ obligaciones en el centro de trabajo ( no se debe a comportamiento negativista o a incapacidad para comprender instrucciones) e) A menudo tiene dificultades para organizar tareas y actividades

f) A menudo evita el disgusto, es renuente a dedicarse en tareas que requieren un esfuerzo mental sostenido (como tra bajos escolares 0 domésticos.

g) A menudo extravía objetos necesarios para tareas 0 actividades (ej. Juguetes, ejercicios escolares, lápices).

h) A menudo se distrae fácilmente por estímulos irrelevantes.

i) A menudo es descuidado en las actividades diarias.

a) A menudo mueve en exceso manos y pies, 0 se remueve en su asiento.

b) A menudo abandona su asiento en clase 0 en otras situaciones donde se espera que permanezca sentado.

c) A menudo corre o salta excesivamente en situaciones en que es inapropiado hacerlo. d) A menudo tiene dificultades para jugar 0 dedicarse tranquilamente a actividades de ocio. e) A menudo "está en marcha" o suele actuar como si tuviera un motor. f) A menudo habla en exceso.

\section{Impulsividad:}

g) A menudo precipita respuestas antes de haber sido completadas las preguntas.

h) A menudo tiene dificultades para guardar el turno.

i) A menudo interrumpe o se inmiscuye en las actividades de otros.

B. Algunos síntomas de hiperactividad - impulsividad o desatención que causaban alteraciones estaban presentes antes de los 7 años de edad.

C. Algunas alteraciones provocadas por los síntomas, se presentan en dos o más ambientes (escuela, trabajo, casa).

D. Debe existir pruebas claras de un deterioro clínicamente significativo de la actividad social, académica o laboral.

E. Los síntomas no a parecen exclusivamente en el transcurso de un trastorno generalizado de desarrollo, esquizofrenia u otro trastorno psicótico, y no se explican mejor por la presencia de otro trastorno mental, (trastorno de estado de ánimo, trastorno de ansiedad, disociativo o de personalidad).

se evidenció diferencias entre los dos grupos de acuerdo al nivel académico al momento de la consulta, a la edad de inicio de los síntomas y a la edad que tenía en la primera asistencia a dicha consulta (tabla 2).

En la tabla 3 se puede apreciar que los pacientes con TDAH tienen con más frecuencia antecedes familiares de este trastorno, así como antecedentes cardiovasculares en sus padres, complicaciones durante el parto, y bajo peso al nacer en comparación con los controles. Adicionalmente, se observó que los casos con TDAH nacieron en su mayoría por cesárea.

El antecedente de PE se presentó en un 3.7\% de los pacientes con TDAH y en un 5.5\% de los controles (OR 0.66, IC95\% 0.34 a 1.29; p=0.218). Esta falta de asociación se mantiene luego de ajustar por género del paciente (OR 3.39, IC95\% 2.31 a 4.98), ser adoptado (OR 1.30, IC95\% 0.29 a 5.71 ), parto por cesárea (OR 0.69, IC95\% 0.48 a 1.00) y edad de inicio de los síntomas (OR 0.28, IC95\% 0.19 a 
Tabla 2. Características de los pacientes estudiados.

\begin{tabular}{lccc}
\hline Característica & $\begin{array}{c}\text { Controles } \\
(\mathbf{n}=404)\end{array}$ & Casos (n=411) & $\begin{array}{c}\text { Valor de } \\
\mathbf{p}\end{array}$ \\
\hline Es varón & $205(50.7 \%)$ & $310(75.4 \%)$ & $<0.001$ \\
\hline Es adoptado & $13(3.2 \%)$ & $30(7.3 \%)$ & 0.012 \\
\hline Nivel académico al consultar & & & \\
Desescolarizado & $3(0.7 \%)$ & $4(1.0 \%)$ & $<0.001$ \\
Especial & $3(0.7 \%)$ & - & \\
Preescolar & $40(9.9 \%)$ & $28(6.8 \%)$ & \\
Primaria & $136(33.7 \%)$ & $184(44.8 \%)$ & \\
Secundaria & $171(42.3 \%)$ & $175(42.6 \%)$ & \\
Universitaria & $28(6.9 \%)$ & $4(1.0 \%)$ & \\
No registrado & $23(5.7 \%)$ & $16(3.9 \%)$ & \\
\hline Edad a la primera consulta & & & \\
Preescolar (<6 años) & $41(10.2 \%)$ & $31(7.5 \%)$ & $<0.001$ \\
Escolar (6-11 años) & $168(41.6 \%)$ & $219(53.3 \%)$ & \\
Pubertad (12-15 años) & $130(32.2 \%)$ & $127(30.9 \%)$ & \\
Adolescencia (>15 años) & $65(16.1 \%)$ & $34(8.3 \%)$ & \\
\hline Edad de inicio de los síntomas & & & \\
"Desde siempre" & $90(22.3 \%)$ & $261(63.5 \%)$ & $<0.001$ \\
3-6 años & $85(21.0 \%)$ & $74(18.0 \%)$ & \\
7-10 años & $87(21.5 \%)$ & $37(9.0 \%)$ & \\
11-17 años & $108(26.7 \%)$ & $30(7.3 \%)$ & \\
No registrado & $34(8.4 \%)$ & $9(2.2 \%)$ & \\
\hline
\end{tabular}

Tabla 3. Antecedentes de los pacientes estudiados.

\begin{tabular}{lccc}
\hline Característica & $\begin{array}{c}\text { Controles } \\
(\mathbf{n}=404)\end{array}$ & Casos $\mathbf{( n = 4 1 1 )}$ & $\begin{array}{c}\text { Valor de } \\
\mathbf{p}\end{array}$ \\
\hline Antecedente de TDAH & & & \\
Hermanos & $6(1.5 \%)$ & $12(2.9 \%)$ & 0.164 \\
Madre & $2(0.5 \%)$ & $37(9.0 \%)$ & $<0.001$ \\
Padre & $6(1.5 \%)$ & $75(18.3 \%)$ & $<0.001$ \\
Familia materna & $8(2.0 \%)$ & $52(12.7 \%)$ & $<0.001$ \\
Familia paterna & $8(2.0 \%)$ & $42(10.2 \%)$ & $<0.001$ \\
\hline Antecedentes cardiovasculares & & & \\
Madre & $15(3.5 \%)$ & $13(1.7 \%)$ & 0.666 \\
Padre & $2(0.5 \%)$ & $9(2.2 \%)$ & 0.036 \\
Otro familiar & $90(22.3 \%)$ & $66(16.1 \%)$ & 0.257 \\
\hline Antecedente embarazo del paciente & & & \\
Parto previo & $53(13.1 \%)$ & $59(14.4 \%)$ & 0.601 \\
Infección & $5(1.2 \%)$ & $8(2.0 \%)$ & 0.419 \\
Hemorragia & $30(7.4 \%)$ & $34(8.3 \%)$ & 0.653 \\
Parto por cesárea & $233(55.2 \%)$ & $203(49.4 \%)$ & 0.048 \\
Complicación al parto & $32(7.9 \%)$ & $48(11.7 \%)$ & 0.071 \\
Preeclampsia & $22(5.5 \%)$ & $15(3.7 \%)$ & 0.218 \\
\hline Problemas neonatales & & & \\
Hipoxia & $6(1.5 \%)$ & $9(2.2 \%)$ & 0.454 \\
Necesitó UCl neonatal & $3(0.7 \%)$ & $5(1.2 \%)$ & 0.493 \\
Necesitó incubadora & $5(1.2 \%)$ & $2(0.5 \%)$ & 0.423 \\
Malformación & $6(1.5 \%)$ & $6(1.5 \%)$ & 0.976 \\
Bajo peso & $1(0.3 \%)$ & $6(1.5 \%)$ & 0.061 \\
Macrosomía & $6(1.5 \%)$ & $4(1.0 \%)$ & 0.825 \\
Dificultad respiratoria & $8(2.0 \%)$ & $11(2.7 \%)$ & 0.510 \\
Ictericia & $5(1.2 \%)$ & $4(1.0 \%)$ & 0.954 \\
\hline & & & \\
\hline & & & \\
& & &
\end{tabular}


0.44). Este modelo se ajustó adecuadamente (48 patrones de covariables; $\chi^{2}=35.11,38$ grados de libertad, $\left.y \mathrm{p}=0.604\right)$.

\section{Discusión}

El TDAH tiene una prevalencia cercana al $10 \%$ a nivel mundial y en nuestro país su prevalencia es aún mayor; ${ }^{4-5}$ donde se resalta la importancia de investigar con el fin de encontrar datos objetivos, que permitan dar una explicación aproximada de qué patologías tienen mayor relación con el trastorno en mención, objetivo del presente artículo. De esta manera, se podría diseñar un método de prevención y seguimiento.

El TDAH es una patología que en promedio se diagnostica en pacientes mayores de 5 años, ${ }^{4-5}$ que concuerda con los resultados encontrados en el presente estudio. El $50 \%$ de los pacientes fueron diagnosticados a una edad mayor a los 6 años; cabe resaltar, que la mayoría de los casos se encuentran cursando principalmente grados escolares de primaria y secundaria. Al momento de definir el inicio de los síntomas, el 63,5\% de los casos, dichos síntomas estaban presentes "Desde siempre", lo cual indica una falla en la búsqueda aún más precoz de síntomas o, tal vez, de antecedentes que sugirieran iniciar la exploración de los síntomas en los niños a una edad más temprana que a la edad promedio encontrada; para así comenzar un tratamiento y asesoramiento familiar adecuado a temprana edad.

Por otra parte, se encontró significativamente que los hijos adoptados con TDAH fueron más prevalentes en los casos que en los controles ( $\mathrm{n}: 30$ (7.3\%) vs n:13 (3.2\%), p 0.012), lo cual permite deducir que, a pesar de no ser un número significativo de muestra con respecto a la total seleccionada, hay una población de la que no se está teniendo clara información acerca de su asociación o antecedente directo con respecto a tener TDAH y ser hijo de madre que haya tenido PE.

En el TDAH a pesar de tenerse relativamente claro el hecho de que un daño directo o cercano al sistema dopaminergico, genera la posterior aparición del trastorno, siendo la hipoxia la forma de daño local más asociada; ${ }^{16,}{ }^{21}$ existe un gran número de patologías que pueden llevar a su desarrollo, impidiendo así concluir una causa clara del trastorno mental a estudio. La gran mayoría de investigaciones en el TDAH se dirigen hacia factores del periodo perinatal; ${ }^{15,22,24-25}$ dentro de ellos, el antecedente de PE materna, poco estudiado; aunque no se evidencio una asociación significativa entre casos y controles, no se puede concluir con certeza que la asociación no esté presente ya sea en forma independiente o en combinación con múltiples factores. Se propone que en futuros estudios se analicen dichos factores, así como lo reportado por Mann. et al, ${ }^{26}$ quienes encontraron una posible asociación entre presentar infección del tracto genitourinario y/o PE, con mayor probabilidad de desarrollar TDAH, aunque recomiendan continuar con futuras investigaciones para describir el mecanismo asociado. $^{18}$

\section{Conclusiones}

En el presente estudio no se encontró la relación del antecedente de PE materno como factor asociado a pacientes con TDAH; sin embargo, no se descarta que la PE no tenga relación con el TDAH; puesto que esta, puede aumentar el riesgo de desarrollar dicho trastorno, y en presencia de otros factores perinatales aún más. ${ }^{26}$

\section{Conflicto de intereses}

Los autores declaran libremente no tener conflicto de intereses.

\section{Referencias}

1. Vera A, Ruano MI, Ramírez LP. Características clínicas y neurobiológicas del trastorno por déficit de la atención e hiperactividad. Colombia Médica. 2007;38:433-9.

2. Cardo E, Nevot A, Redondo M, Melero A, de Azua B, Garcia-De la Banda G, et al. Attention deficit disorder and hyperactivity: a pattern of evolution? Revista de neurologia. 2010;50 Suppl 3:S143-7. Epub 2010/03/05. Trastorno por déficit de atencion/hiperactividad: un patron evolutivo?

3. Grupo de expertos nacionales para el estudio del trastorno por deficit de atención e hiperactividad GENPETDAH AC. 1er Consenso Latinoamericano sobre el trastorno por deficit de atención e hiperactividad; México. 2007.

4. Pineda D, Ardila A, Rosselli M, Arias BE, Henao GC, Gomez LF, et al. Prevalence of attentiondeficit/hyperactivity disorder symptoms in 4- to 17-yearold children in the general population. Journal of abnormal child psychology. 1999;27(6):455-62. Epub 2000/05/23.

5. Cornejo JW, Osío O, Sánchez Y, Carrizosa J, Sánchez $\mathrm{G}$, Grisales H, et al. Prevalencia del trastorno por déficit de atención-hiperactividad en niños y adolescentes colombianos. Rev Neurol. 2005;40:716-22.

6. Getahun D, Jacobsen SJ, Fassett MJ, Chen W, Demissie K, Rhoads GG. Recent trends in childhood attention-deficit/hyperactivity disorder. JAMA pediatrics. 2013;167(3):282-8. Epub 2013/01/23.

7. St Sauver JL, Barbaresi WJ, Katusic SK, Colligan RC, Weaver AL, Jacobsen SJ. Early life risk factors for attention-deficit/hyperactivity disorder: a populationbased cohort study. Mayo Clinic proceedings Mayo Clinic. 2004;79(9):1124-31. Epub 2004/09/11.

8. Lou HC. Etiology and pathogenesis of attention-deficit hyperactivity disorder (ADHD): significance of prematurity and perinatal hypoxic-haemodynamic encephalopathy. Acta Paediatr. 1996;85(11):1266-71. Epub 1996/11/01.

9. Capdevila C, Navarro JB, Artigas J, Obiols J. Complicaciones obstétricas y médicas en el Trastorno de 
Déficit Atencional/Hiperactividad (TDAH): ¿hay diferencias entre los subtipos? International Journal of Clinical and Health Psychology. 2007;7:679-95.

10. Halmoy A, Klungsoyr K, Skjaerven R, Haavik J. Pre- and perinatal risk factors in adults with attentiondeficit/hyperactivity disorder. Biological psychiatry. 2012;71(5):474-81. Epub 2011/12/28.

11. Castellanos Castellanos YA, Moreno Castañeda NF, Villamizar Caballero HY, Gamboa Blanco LM, Sus Carrizosa SE, Guio Mahecha E. Variables pre-analíticas que afectan la cuantificación sérica de Proteína-CReactiva e IL-6 en primigestantes preeclámpticas y controles sanos. Revista de la Universidad Industrial de Santander Salud. 2012;44:25-30.

12. Firoz T, Sanghvi H, Merialdi M, von Dadelszen P. Preeclampsia in low and middle income countries. Best practice \& research Clinical obstetrics \& gynaecology. 2011;25(4):537-48.

13. Martin JN, Jr., Brewer JM, Wallace K, Sunesara I, Canizaro A, Blake PG, et al. Hellp syndrome and composite major maternal morbidity: importance of Mississippi classification system. The journal of maternal-fetal \& neonatal medicine : the official journal of the European Association of Perinatal Medicine, the Federation of Asia and Oceania Perinatal Societies, the International Society of Perinatal Obstet. 2013;26(12):1201-6.

14. Bramham K, Parnell B, Nelson-Piercy C, Seed PT, Poston L, Chappell LC. Chronic hypertension and pregnancy outcomes: systematic review and metaanalysis. Bmj. 2014;348:g2301.

15. Zappitelli M, Pinto T, Grizenko N. Pre-, peri-, and postnatal trauma in subjects with attention-deficit hyperactivity disorder. Canadian journal of psychiatry Revue canadienne de psychiatrie. 2001;46(6):542-8. Epub 2001/08/31.

16. Fernández M, Hidalgo I, Lora A, Sánchez L, Rodríguez L, Lefa E, et al. Consenso multidisciplinar y evidencias para la puesta en práctica. La atención primaria y el trastorno por déficit de atención con/sin hiperactividad (TDAH): ACINDES; 2009.

17. Getahun D, Rhoads GG, Demissie K, Lu SE, Quinn VP, Fassett MJ, et al. In utero exposure to ischemic-hypoxic conditions and attention-deficit/hyperactivity disorder.
Pediatrics. 2013;131(1):e53-61. Epub 2012/12/12.

18. Rodriguez A, Miettunen J, Henriksen TB, Olsen J, Obel $\mathrm{C}$, Taanila A, et al. Maternal adiposity prior to pregnancy is associated with ADHD symptoms in offspring: evidence from three prospective pregnancy cohorts. Int $\mathrm{J}$ Obes (Lond). 2008;32(3):550-7. Epub 2007/10/17.

19. du Prel Carroll X, Yi H, Liang Y, Pang K, Leeper-Woodford $S$, Riccardi $\mathrm{P}$, et al. Family-Environmental Factors Associated with Attention Deficit Hyperactivity Disorder in Chinese Children: A Case-Control Study. PLoS ONE. 2012;7(11):e50543.

20. Mick E, Biederman J, Faraone SV, Sayer J, Kleinman S. Case-control study of attention-deficit hyperactivity disorder and maternal smoking, alcohol use, and drug use during pregnancy. Journal of the American Academy of Child and Adolescent Psychiatry. 2002;41(4):378-85. Epub 2002/04/05.

21. Kidd PM. Attention deficit/hyperactivity disorder (ADHD) in children: rationale for its integrative management. Alternative medicine review : a journal of clinical therapeutic. 2000;5(5):402-28. Epub 2000/11/01.

22. Banerjee TD, Middleton F, Faraone SV. Environmental risk factors for attention-deficit hyperactivity disorder. Acta Paediatr. 2007;96(9):1269-74. Epub 2007/08/28.

23. Itami-Sordo ME, Jiménez-Nieto R, de Haro R. Factores vasculares implicados en la preeclampsia. Revista de la Facultad de Medicina (México). 2013;56:18-24.

24. Pringsheim T, Sandor P, Lang A, Shah P, O'Connor P. Prenatal and perinatal morbidity in children with Tourette syndrome and attention-deficit hyperactivity disorder. Journal of developmental and behavioral pediatrics : JDBP. 2009;30(2):115-21. Epub 2009/03/27.

25. Ray GT, Croen LA, Habel LA. Mothers of children diagnosed with attention-deficit/hyperactivity disorder: health conditions and medical care utilization in periods before and after birth of the child. Medical care. 2009;47(1):105-14. Epub 2008/12/25.

26. Mann JR, McDermott S. Are maternal genitourinary infection and pre-eclampsia associated with ADHD in school-aged children? Journal of attention disorders. 2011;15(8):667-73. Epub 2010/09/15. 